\title{
El problema iatrogénico del dietiletilbestrol. DES.
}

\author{
Dr. Alberto Duarte-Contreras*
}

En 1938 se realizó la síntesis del dietiletilbestrol, DES. Poco después se inició su administración por vía oral para el tratamiento del embarazo de alto riesgo, dados los efectos benéficos logrados por Smith y colaboradores en pacientes con preeclampsia, eclampsia, aborto habitual, parto prematuro, diabetes, hipertensión y muerte fetal in útero. Se daban de 5 a $150 \mathrm{mg}$. día a partir de la semana 12 de gestación, hasta la semana 35 , con una dosis total hasta de $12.200 \mathrm{mg}$ ( $(7,9)$.

En 1953 Dickman y colaboradores, al realizar un estudio prospectivo controlado doble ciego, en 840 embarazadas que recibían DES, concluyeron que no tenía efecto alguno benéfico en el tratamiento del embarazo de al to riesgo (8). Con todo, se siguió administrando con igual entusiasmo. En 1971 Herbst y colaboradores informaron haber encontrado adenocarcinoma de células claras en la vagina de 7 jóvenes cuyas edades oscilaban entre 14 y 22 años y se preguntaban qué relación pudiese tener este extraño cáncer de vagina

* Del Departamento Gineco Obstétrico del Hospital San Juan de Dios. Cúcuta. y cérvix en la exposición al DES a la que habían sido sometidas $(11,20)$.

En 1971 la FDA proscribió el uso del DES en mujeres embarazadas; con todo, se siguió empleando por un tiempo más en diferentes países. En Estados Unidos más de tres millones de embarazadas fueron expuestas al DES entre 1945 y $1957(\%)$.

Dado el al to número de alteraciones en el epitelio vaginal, adenosis, que se observó en pacientes jóvenes expuestas, fue necesario constituir la DESAD, una Asociación para velar por las jóvenes expuestas al DES (22), y se acordó a su vez adelantar un seguimiento para establecer si estas lesiones se encuentran también en mujeres expuestas, con edad superior a los 24 años. Hoy se sabe que el problema del DES es simplemente un problema de iatrogenicidad.

Hacia la décima semana de vida intrauterina toda la vagina se encuentra cubierta por un epitelio columnar cuboidal simple de origen mülleriano y es reemplazado por epitelio escamoso del seno urogenital; es en este momento cuando se presenta la interferencia del DES. 
Se ha establecido que en las jóvenes de 7 a 24 años que fueron expuestas, el factor de riesgo va de 1.4 por mil a 1.4 por diez mil (16).

En nuestro país, a pesar del empleo profuso que tuvo el esteroide sintético, no se ha informado el hallazgo de adenocarcinoma de células claras de vagina y cérvix en jóvenes expuestas. Nosotros hemos tenido oportunidad de hacer un riguroso seguimiento por petición de las madres a quienes administramos DES en su embarazo, a cinco jóvenes que pasaron ya los 24 años y no observamos lesión alguna benigna ni maligna en vagina y cérvix. Con todo, debemos permanecer alerta siempre que veamos una niña mayor de 7 años que hubiese sido expuesta in útero a la acción del DES.

\section{EFECTOS ADVERSOS}

Son múltiples los efectos adversos que pueden presentar las adolescentes expuestas al DES, benignos unos, malignos otros (30).

Entre los efectos transplacentarios benignos se encuentran lesiones sobre el epitelio vaginal, el cuello uterino, el útero $y$ las trompas, a más de alteraciones en la función menstrual y reproductiva $(4,10,25$, 29), lesiones todas que se pueden observar en las no expuestas (15).

Sobre el epitelio vaginal se puede encontrar la adenosis.

Entre las anomalías anatómicas se han descrito collares, capuchones, septum, crestas cérvico vaginales, estenosis cervical, cuello incompetente, cérvix hipoplásico, cavidad uterina pequeña o en forma de $T$, o con bandas de constricción, sinequias y pólipos. El más frecuente es el reborde cérvico vaginal que se encuentra entre el 22 y el $58 \%$ de las pacientes $(4,13)$. El diagnóstico de estas anomalías se hace por visión directa con vaginoscopio o con espéculo en algunos casos y con rayos $X$ en otros.

Entre las anormalidades reproductivas se ha comprobado esterilidad, si bien el problema principal radica en pérdidas fetales por aborto espontáneo, embarazo ectópico, parto prematuro, muerte fetal y aún muerte neonatal $(2,3,27)$. Sin embargo, otros estudios muestran que el $81 \%$ de las expuestas que han quedado embarazadas han tenido partos a término con productos vivos (23).

Se presentan también problemas sicológicos serios tanto en las madres que recibieron el DES como en sus productos femeninos cuando llegan a la adolescencia (26); tal es el caso de nuestras cinco pacientes estudiadas.

Como efecto maligno se ha relacionado el hallazgo de adenocarcinoma de células claras de vagina y cérvix. Algunos autores creen que hay un aumento significativo de cáncer mamario en las expuestas (5), pero investigaciones recientes no han podido confirmar la veracidad de esta información.

Las anomalías benignas se presentan con mayor frecuencia que el adenocarcinoma de células claras.

\section{ADENOSIS}

La adenosis es la presencia anormal de epitelio glandular en la vagina, o sea. la presencia de un tejido extraño que se ha introducido anormalmente dentro del tejido pavimentoso poliestratificado (1); estas estructuras glandulares se colocan en la superficie y entran en contacto íntimo con el tejido epitelial. La adenosis no es un tejido canceroso ni precursor del adenocarcinoma de células claras pero sí es causante de serias preocupaciones. Con el correr del tiem.po, el epitelio cilíndrico de la adenosis es substituido por metaplasia 
plana. No se ha visto el primer caso en el que progrese a carcinoma de células cla$\operatorname{ras}(17,18)$.

La adenosis se encuentra en el 35 al $90 \%$ de las expuestas al DES, según unos autores. Nosotros jamás la hemos observado, a pesar de haberla buscado con insistencia ya que tenemos conciencia de la liberalidad con que se administró el DES a embarazadas en nuestro medio durante unas tres décadas. Se ha observado que en un buen número de pacientes desaparece de manera espontánea entre los 22 y los 23 años de edad (1).

Cuando la adenosis no ha sufrido transformación metaplásica, se reconoce con cierta facilidad por su aspecto rojizo y granuloso, como una estructura en racimo de uvas con pequeños quistes y lesiones papilares que parecen tener localización multicéntrica. Estas áreas no toman el lugol. Toda zona de color rojo intenso en adolescentes expuestas hace sospechar un carcinoma de células claras.

\section{ADENOCARCINOMA DE CELULAS CLARAS DE VAGINA Y CERVIX}

En 1971 el Registro de Adenocarcinoma de células claras en pacientes jóvenes había recopilado 300 casos en los Estados Unidos, de los cuales alrededor del $60 \%$ correspondían a pacientes expuestas in útero a la acción del DES (12).

La incidencia en jóvenes expuestas menores de 24 años va del 1 al 4 por mil, siendo una niña de 7 años la menor edad.

Este carcinoma se conoció mucho antes de que se hubiese realizado la síntesis del dietiletilbestrol; ello quiere decir que en su génesis hay otros factores diferentes al DES $(15,20)$.

Herbs registró en 1974 ciento setenta casos: 100 en vagina y 70 en cérvix de mujeres entre 7 y 24 años(14).
El síntoma llamativo es un sangrado genital por lo general escaso, o una secreción sanguinolenta persistente. Puede involucionar también de manera silenciosa $y$ entonces el diagnóstico es un hallazgo de examen. Para reconocerlo se requiere visión directa de la lesión sea por vaginoscopía o por especuloscopía.

Puede localizarse en vagina, en cérvix, - simultáneamente en vagina y cérvix, Cuando se localiza en vagina, en el $70 \%$ se le encuentra sobre la pared anterior, en el $20 \%$ sobre la pared posterior y en el $10 \%$ sobre las paredes laterales.

Las lesiones son de color rojo intenso y tienen diferente forma y tamaño: como masas poliposas de base sólida, o alargadas de aspecto friable y fungoso, con zonas ulceradas (31). Las lesiones vaginales casi siempre son de escasa profundidad, no así las cervicales.

La malignidad no depende de la dosis de DES que se haya recibido y obedece más a la actividad mitótica que al tamaño de la neoplasia. Cuando la localización es cervical se encuentra un mayor número de metástasis.

Histológicamente la lesión está constituida por células cilíndricas y cuboides, algunas aplanadas, de diferente tamaño, que en ocasiones forman túbulos o quistes cuya superficie se tapiza por células en herradura, con proyecciones intraluminares a manera de papilas. En algunas ocasiones las células epiteliales se agrupan en forma de nidos o de masas sólidas. El citoplasma de las células en herraduras y la mayoría de las epiteliales es rico en glucógeno y por ello tienen un aspecto claro: de ahí el nombre que reciben de "células claras".

\section{CONDUCTA}

A toda adolescente expuesta in útero a la acción del DES se le debe explorar con 
detenimiento su aparato reproductor después de la menarca, así sea asintomática. Otro tanto se hará con las expuestas que a los 15 años no hayan menstruado o que estando embarazadas consulten por amenaza de aborto.

Cuando adolescentes expuestas presenten síntomas sugestivos de tumor vaginal o cervical, tales como un sangrado anormal, el examen se debe practicar de inmediato y mejor bajo anestesia general. Si no se encuentra lesión alguna, el resultado no se puede considerar como definitivo; se repite el examen cada año hasta cuando la joven alcance la edad de 20 a 22 años. Si se observan áreas de adenosis, se practican exámenes más frecuentes y e..tonces se hace indispensable la colposcopía.

El examen consiste en inspección de los genitales externos, visualización de las paredes vaginales con vaginoscopio o con espéculo, tacto vaginal o palpación rectoabdominal, frotis para citología tumoral, test de Schiller, más colposcopía y biopsia cuando estén indicadas.

La palpación de las paredes vaginales debe ser cuidadosa y practicada sobre toda el área, incluyendo los fondos de saco ya que éstos, cuando hay adenosis, se encogen y aparecen más pequeños y menos elásticos que lo normal; es el único método que nos puede informar con seguridad la presencia de zonas induradas $(\overline{6})$. Por lo general la inspección y la palpación son más importantes que la prueba o test de Schiller.

Cuando tanto las paredes vaginales como el cérvix se ven con nitidez, es fácil detectar las anomalías anatómicas del cuello (collares, surcos circulares, rebordes transversos, hiperplasia y otras anormalidades menos frecuentes).

Cuando la adenosis no ha sufrido ansformación metaplásica, se le reco- noce por su aspecto rojizo y granuloso, con pequeños quistes y lesiones papilares que parecen tener localización multicéntrica. Estas áreas no toman el lugol. Cuando la zona tiene un color rojizo intenso muy posiblemente es asiento de un adenocarcinoma de células claras.

La citología puede mostrar cambios importantes del epitelio vaginal y cervical. Para la toma de muestras, se debe eliminar primero el exceso de moco o de secreciones haciendo un aseo suave con solución salina; luego, con bajalenguas diferentes, se toma un frotis de cada uno de los cuatro costados en el tercio inferior, tercio medio y tercio superior de la vagina y luego del ectocérvix y del endocérvix; se hacen los extendidos sobre láminas marcadas (v.g. tercio medio cara anterior, posterior, lateral derecha, lateral izquierda), se fijan en alcohol etílico al 95\% y se colorean con el Shorr o con el Papanicolaou (32).

Cuando no hay colposcopio la extensión de las lesiones se reconoce con la prueba de Schiller.

Algunos ginecólogos practican la colposcopía de rutina en el primer examen; es suficiente, sin que haya necesidad de repetirla en exámenes posteriores puesto que sólo se hace indispensable cuando la citología indica malignidad: sirve entonces para orientar la toma de las muestras que se van a remitir a estudio histológico (32). La colposcopía como rutina, nunca es superior a la citología ni a la pal pación y sólo colposcopistas expertos pueden reconocer la proliferación de la metaplasia o la presencia de displasia (28). La colposcopía tampoco detecta un carcinoma de células claras insospechado.

La biopsia se toma sólo cuando lo in. dique la citología, o cuando se aprecien nódulos sospechosos, o cuando en un control se constate aumento del tamaño o de la friabilidad de los tejidos. 
No es necesario practicar histerosalpingografía a todas las adolescentes que fueron expuestas al DES para descubrir posibles alteraciones anatómicas: se ordenará sólo a quienes presenten problemas de fertilidad v.g. aborto habitual (27).

El cerclaje se practica únicamente cuando haya signos de dilatación o de incompetencia cervical.

Quienes han sido expuestas al DES pueden usar contraceptivos hormonales $y$ corren un riesgo similar al de las no expuestas.

Cuando hay deformidades de cérvix - cuando los tejidos de los fondos de saco han perdido su elasticidad o son fibrosos, es muy difícil adaptar diafragmas. Kaufman ha encontrado dificultad para la aplicación de dispositivos intrauterinos a algunas pacientes por cambios o alteraciones morfológicas endocervicales (1).

Algunos autores creen que las adolescentes expuestas corren los mismos riesgos de sufrir lesiones escamosas que las no expuestas (1).

La adenosis sólo requiere tratamiento cuando se acompaña de una ectopia marcada que se constituya en peligro de ocasionar sangrado copioso. Se trata aplicando óvulos de progesterona mas criocirugía o rayos laser. Se debe controlar luego con biopsias (1). Es innecesaria la extirpación quirúrgica y más aún la vaginectomía y la radioterapia.

El tratamiento del adenocarcinoma de células claras es radical: histerectomía con vaginectomía más linfadenectomía pélvica ya que en el $17 \%$ de las lesiones vaginales en estado I y en el $28 \%$ de las lesiones cervicales estados II y Ila se encuentran nódulos. Cuando el diagnóstico cs precoz, los resultados son halagadores (24).
Cuando el diagnóstico es tardío se requiere practicar exenteración con implantación de uréteres en la bolsa ileal. Ultimamente se ha empleado la quimioterapia con resultados satisfactorios.

La recidiva o la muerte antes de los tres años se encuentra en el $24 \%$ de los casos (24). El 50\% de las metástasis se encuentran en la pelvis, en los ganglios supraclaviculares y en los pulmones (24).

Como en las últimas cuatro décadas se ha detectado este carcinoma mesonefroide, es indispensable buscarlo en toda niña mayor de 7 años que presente sangrado anormal o secreción sanguinolenta vaginal, así no haya la seguridad de que recibió el DES durante su vida intrauterina. Cuando se confirma el antecedente de la ingestión del DES, aún en jóvenes asintomáticas, se hace caso omiso de la dosis recibida que no se considera como factor determinante, y se inicia el estudio ginecológico detenido.

\section{RESUMEN}

Desde inicios de la cuarta década del presente siglo se empleó el dietiletilbestrol, DES, para el tratamiento del embarazo de alto riesgo. Millones de embarazadas fueron tratadas. Treinta años más tarde se comprobó que el esteroide sintético no proporcionaba beneficio alguno a estas pacientes y que al contrario un buen número de jóvenes de 7 a 24 años que habían sido expuestas in útero a la acción del DES presentaban adenocarcinoma de células claras de vagina y cérvix. Se proscribió su empleo en 1971. Con todo en muchos lugares se le siguió prescribiendo por más años. En las jóvenes expuestas se han encontrado lesiones benignas y malignas. Entre las lesiones benignas figuran al teraciones histológicas en el epitelio vaginal (adenosis), anatómicas en útero y trompas, a más de alteraciones de la función menstrual y reproductiva. 
Se estudian la adenosis y el adenocarcinoma de células claras, enfatizando sobre la manera de hacer el seguimiento a partir de los 7 años a toda joven que hubiese sido expuesta al DES y sobre la metodología del examen tanto a sintomáticas como a asintomáticas. La adenosis no requiere tratamiento quirúrgico, en tanto que en el adenocarcinoma de células claras es indispensable la cirugía radical (histerec-

\section{BIBLIOGRAFIA}

1. BARBER, H.R.K. Vaginal adenosis. En: Quilligan, E. J. Current therapy in Obstetrics and Gynecology. W.B. Saunders, Co. Philadelphia. 144, 1983.

2. BARNES, A. B, COLTON, T., GUNDERSEN, J, y cols. Fertilidad y resultado del embarazo en mujeres expuestas in útero al dietilestilbestrol. N. Eng. J. Med. 302: 609, 1980.

3. BERGER, M. J y GOLDSTEIN, D. $P$. Impaired reproductive perfomance in DES exposed women. Obstet. Gynecol, 55: 25. 1980.

4. BIBBO, M., GILL, W. B., AZIZI, F. y cols. Follow-up study of male and female offspring of DES-exposed mothers. Obstet. Gynecol. 49: 1, 1977.

5. BIBBO, M., HAENSZEL, W. M., WIED, G. L. y cols. A twenty-five follow-up study of women exposed to diethylstilbestrol during pregnancy. N. Engl. J. Med. 298: 763, 1978.

6. BIBBO, M. Screening of individuals exposed to diethylstilbestrol. Clin. Obstet. Gynecol, 22: 689, 1979.

7. BIBBO, M. y GILL, W. B. Investigación de adolescentes expuestas al dietiletilbestrol durante la vida intrauterina. Clin. Pediátr. N. Am. 2: 377, 1981. tomía, vaginectomía más linfadenectomía pélvica y aún exenteración en casos avanzados). Se ha empleado también la quimioterapia. Con diagnóstico precoz el pronóstico es menos sombrío que cuando se trata tardíamente.

Hicimos seguimiento a cinco paciente, expuestas, asintomáticas, sin que hubiésemos observado alteración alguna.

8. DIECKMANN, W.J., DAVIS, M. E., RYNKIEWCZ, L.M. y cols. Does the administration of diethylstilbestrol during pregnancy have therapeutic value? Am. J. Obst. Gynec. 66:1062, 1953.

9. HEINONEN, O. P. Diethylstilbestrol in pregnancy: frecuancy of exposure and usage patterns. Cancer. 31: 573, 1973.

10. HANEY, A. F., HAMMOND, C. B., SOULES, M. R. y cols. Diethylstilbestrol-induced upper genital tract abnormalities. Fertil. Steril, 31: 142, 1979 .

11. HERBST, A. L., ULFELDER, H. y POSKANZER, D. C. Adenocarcinoma of the vagina: association of maternal stilbestrol therapy with tumor appearance in young women. N. Engl. J. Mèd. 284: 878, 1971.

12. HERBST, A. L., KURMAN, R. J., SCULLY, R. E. y cols. Clear-cell adenocarcinoma of genital tract in young female: Registry report. N. Engl. J. Med. 287: 1259, 1972 .

13. HERBST, A. L. y cols. Stilbestrol induced abnormalities of the genital tract in young women. En: Progress in gynecology. Taymor, M. y Green, T. Ed. New York. Grund and Stratton. Vol. 6, 1975. 
14. HERBST, A., ROBBOY, S. SCULLY, R. y POSKANZER, D. Clear- cell adenocarcinoma of the vagina and cervix girls. Analysis of 170 registry cases. Am. J. Obst. Gynec. 119: 713, 1974.

15. HERBST, A. L., POSKANZER, D.C., ROBBOY, S. J. y cols. Prenatal exposure to stilbestrol: a prospective comparison of exposed female offspring with inexposed controls. N. Engl. J. Med. 292: 334, 1975.

16. HERBST, A.L., COLE, P., COLTON, $T$. y cols, Age-incidence and risk of diethylstilbestrol-related clear cell adenocarcinoma of the vagina and cervix. Am. J. Obst. Gynec. 128: 43. 1977.

17. Ng, A.B.P., REAGAN, J.W., JAWLICZAK, S. y cols. Cellular detection of vagina adenosis. Obstet. Gynecol. 46: 323,1975 .

18. NG, A.B.P., REAGAN, J.W., NADJI, $M$ y cols. Natural history of vaginal adenosis in women exposed to diethylstilbestrol in utero. J. Reprod. Med. 18: 1, 1977.

19. NOLLER, K.L., DECKER, D.C., LANIER, A.P. y cols. Clear cell adenocarcinoma of the cervix after maternal treatment with synthetic estrogens. Mayo Clin.Proc. 47: 629, 1972.

20. NOLLER, K.L. y FISK, C.R. Diethylstilbestrol. usage: its interesting past, important present and questionable future. Med. Clin. North Am. 58: 793,1974 .

21. NOLLER, K.L., DECKER, D.G., SYMMONS, E.R., DOCKERTY, M.B, y KURLAND, L.T. Clear-cell adenocarcinoma of the vagina and cervix. Survival data. Am. J. Obst Gynec. 124: 285, 1976.

22. O'BRIEN, P.C., NOLLER, K.L., ROBBOY, S.J., BARNES, A.B. y cols. Alteraciones del epitelio vaginal en mujeres jóvenes inscritas en el Pro- yecto Cooperativo Nacional sobre Adenosis por Dietiletilbestrol (DESAD). Obstet. Gynecol. 53: 300, 1979 .

23. RICHMOND, J.B. Physisian advisory: Health effects of the pregnancy use of diethylstilbestrol. Washingtong, D. C. Department of Health, Education and Welfare. October 4, 1978.

24. ROBBOY, S., HERBST, A.L. y SCULLY, R.E. Clear-cell adenocarcinoma of the genital tract in young females. Analysis of 37 tumors that persisted or recurred after primary therapy. Cancer. 34: 606, 1974.

25. SNADBERG, E.C. Bening cervical and vaginal changes associated with exposure to stilbestrol in utero. Am. J. Obst. Gynec. 125: 777, 1976.

26. SCHWARTZ, R.W. y STEWART, N.B. Efectos sicológicos de la exposición al dietiletilbestrol. J.A.M.A. 252,1977 .

27. SIEGLER, A.M., WANG, C.F., FRIBERG, P. Fertility of the diethylstilbestrol-exposed offspring. Fertil. Steril. 31: 601, 1979.

28. STAFL, A. Colposcopy in evaluation of DES-exposed women. En: Herbst, A.L. (ed): Intrauterine exposure to diethylstilbestrol in the human. Proceedings of Symposium of DES. 1977. Chicago, American College of Obstetricians and Gynecologists 1978.

29. STILLMAN, R.J. In utero exposure to diethylstilbestrol: adverse effects on the reproductive tract and reproduction perfomance in male and female offspring. Am. J. Obst. Gynec. 142: $905,1982$.

30. SCULLY, R. y cols. Vaginal and cervical abnormalities including clear-cell adenocarcinoma related to prenatal exposure to stilbestrol. Am. Clin. Lab. Sci. 4: 222, 1974. 
31. TSUKADA, Y., HWETT, W., BARLOW, J. y PECKREN. J.W, Clear-cell adenocarcinoma (mesonephroma) of the vagina. Cancer. 29: 1208, 1972.

32. TOWNSEND, D.E. Techniques of examination and screening of the-DES- exposed female. En: Herbst, A.L. (Ed): Intrauterine exposure to diethylbestrol in the human. Proceedings of Symposium on DES. 1977. Chicago. American College of Obstetrician and Gynecologists. 1978. 